CrossMark \&click for updates

Cite this: Chem. Commun., 2015, 51,17140

Received 31st July 2015,

Accepted 29th September 2015

DOI: $10.1039 / \mathrm{c} 5 \mathrm{cc} 06415 \mathrm{e}$

www.rsc.org/chemcomm

\title{
Investigation of the interaction modes between nonpolar organic pollutants with ionizable functional groups and natural organic matter via AuNP-based colorimetric assays $\dagger$
}

\author{
Hongyun Niu, ${ }^{a}$ Saihua Wang, ${ }^{a}$ Yixin $\operatorname{Tan}^{\mathrm{ab}}$ and Yaqi Cai*a
}

For the first time, natural organic matter (NOM) modified AuNPs have been used as sensors to "observe" the specific interactions (such as hydrogen-bonds and halogen-bonds) between functional groups of organic compounds and NOM using colorimetric assays.

Nonpolar and moderately polar organic compounds with ionizable functional groups (such as hydroxyl- and amino-substituted aromatics) are usually environmentally significant. ${ }^{1}$ Their binding on dissolved natural organic matter (NOM) directly influences their bioavailability, fate, and behavior in aquatic ecosystems., ${ }^{2,3}$ It is necessary to investigate the affinity ability and binding mechanisms of these kinds of contaminants on NOM, which is usually carried out by measuring the partition coefficient $\left(K_{\mathrm{DOC}}\right)$ through adsorption isotherms of organic pollutants on humic substance. However, the adsorption isotherms are insufficient to estimate the interactions between NOM and the functional groups of nonpolar and moderately polar organic compounds. It is vital to introduce a new method to probe the interaction mechanisms between these chemicals and NOM.

AuNP-based colorimetric assays are of particular interest because molecular events are easily transformed into color changes, which can be observed by the naked eye. The color change is highly sensitive to the size, shape, capping agents, and medium refractive index, as well as the aggregation state of AuNPs. Most assays designed for compounds rely on the binding of ligands modified on AuNPs with target analytes, which is defined as "interparticle crosslinking aggregation". 4 In this study, for the first time, we have used NOM modified AuNPs as sensors to investigate the interactions between organic contaminants and NOM through a color change of AuNP suspension. According to the results of colorimetric

\footnotetext{
${ }^{a}$ State Key Laboratory of Environmental Chemistry and Ecotoxicology, Research Center for Eco-Environmental Sciences, Chinese Academy of Sciences, P.O. Box 2871, Beijing 100085, China. E-mail: caiyaqi@rcees.ac.cn; Tel: +86-10-62849182 ${ }^{b}$ Department of Resources Environmental and Chemical Engineering of Nanchang University, Jiangxi, Nanchang Province, 330031, China

$\dagger$ Electronic supplementary information (ESI) available: Experimental details and additional figures and tables. See DOI: 10.1039/c5cc06415e
}

assays, we then infer backward the interaction modes between NOM and organic targets.

The AuNPs are obtained by reduction of $\mathrm{HAuCl}_{4}(0.025 \mathrm{mM})$ by $\mathrm{NaBH}_{4}$ in the presence of the stabilizer Suwannee River fulvic acid (SRF) (15 mg L $\left.{ }^{-1}\right)$. Detailed information about the synthesis and characterization of SRF-AuNPs is presented in the ESI. $\dagger$ The synthesized SRF-AuNPs are about $5 \mathrm{~nm}$ indiameter and well-dispersed in neutral and alkaline solutions (Fig. 1A and Fig. S1C, ESI $\dagger$ ). According to the study of Huo, ${ }^{5}$ the extinction coefficient $\varepsilon$ of SRF-AuNP solution is calculated to be $1.03 \times 10^{7} \mathrm{M}^{-1} \mathrm{~cm}^{-1}$. The absorbance of the as-prepared SRF-AuNP suspension at $\lambda=514 \mathrm{~nm}$ is $0.51(\mathrm{RSD}=5 \%)$; therefore, the molar concentration of SRF-AuNP solution is $4.95 \times 10^{-8} \mathrm{~mol} \mathrm{~L}^{-1}$. The elemental composition on the surface of SRF-AuNP sensors is investigated using XPS. Fig. S1E (ESI $\dagger$ ) shows that SRF-AuNPs mainly consist of $\mathrm{C}, \mathrm{O}$, and $\mathrm{Au}$ atoms. The high resolution $O$ 1s XPS line of sensors shows that phenolic groups/ester, carboxylic groups, carbonyl groups are the main oxygen-containing species on the surface of sensors (Fig. S1F, ESI $\dagger$ ). Due to the intra- and intermolecular H-bonding among these oxygen-containing groups in acidic solutions, the SRF-AuNPs show a slight aggregation at pH 3.5-5.5 (Fig. S1A, B and $\mathrm{D}, \mathrm{ESI} \dagger)$. In the presence of monovalent cations $\left(\mathrm{Na}^{+}\right)$or at different temperatures, the dispersion of the sensors remains unchanged (Fig. S2A, ESI $\dagger$ ). However, the addition of 50 and $100 \mu \mathrm{M}$ of $\mathrm{Ca}^{2+}$ leads to aggregation of sensors in the whole $\mathrm{pH}$ range because $\mathrm{Ca}^{2+}$ can bind with the carboxylic or phenolic

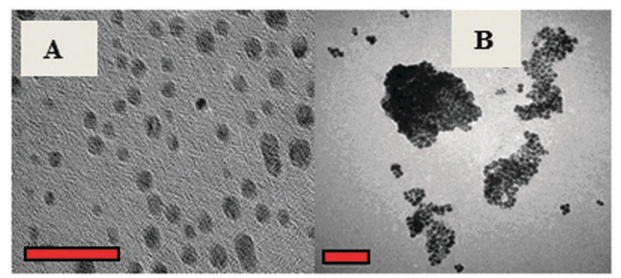

Fig. 1 TME images of the as-prepared SRF-AuNP sensors (A) and sensors in the presence of $\mathrm{PBP}(\mathrm{B})$ at $\mathrm{pH} 7.5$. 
groups of NOM. In the UV-Vis spectra, the location of surface plasmon resonance (SPR) of sensor suspension at $\mathrm{pH}$ 5.5-9.5 shifts from $514 \mathrm{~nm}$ to $530-534 \mathrm{~nm}$ (Fig. S2B-D, ESI $\dagger$ ). Anyway, the as-prepared SRF-AuNP sensors are stable with and without $\mathrm{Ca}^{2+}$, and the color and location of SPR of sensor suspension do not change for several months. We measure the concentrations of residual NOM in a mixture using a fluorescence spectrophotometer according to the fluorescence intensity at Ex/Em = $340 \mathrm{~nm} / 460 \mathrm{~nm}$. As a result, about $5 \mathrm{mg} \mathrm{L}^{-1}$ of free NOM remain in AuNP colloid. The unbound NOM can enhance the solubility of hydrophobic chemicals and reduce the particle-particle interaction between AuNPs. ${ }^{6}$

The SRF-AuNP sensors are used to investigate the interaction modes between NOM and several highly hydrophobic and moderately hydrophobic phenols, bisphenol A (BPA), tetrabromobisphenol A (TBBPA), pentabromophenol (PBP), pentachlorophenol (PCP), and tetrachlorobisphenol A (TCBPA). As a result, only TBBPA and PBP trigger the aggregation of SRF-AuNP sensors at $\mathrm{pH} \leq 5.5$, a notable color change and red-shift of SPR peaks in UV-Vis spectra of sensor suspension are seen in Fig. S3 and $\mathrm{S} 4$ (ESI $\dagger$ ). At $\mathrm{pH}$ 7.5-9.5, the two targets do not cause aggregation of sensors. The SRF-AuNP sensors show no response to the chlorinated phenols and BPA across the $\mathrm{pH}$ range.

The influence of coexisting cations $\left(\mathrm{Na}^{+}\right.$and $\left.\mathrm{Ca}^{2+}\right)$ on the interactions between targets and NOM can be explored using the NOM-AuNP sensors as well. The presence of 0.2, 1.0 and $10 \mathrm{mM}$ of $\mathrm{Na}^{+}$does not affect the response of sensors to these compounds (Fig. S5-S7, ESI $\dagger$ ). The addition of $\mathrm{Ca}^{2+}$ cations $(5,10,50$ and $100 \mu \mathrm{M})$ has no influence on the response of PCP and TCBPA to SRF-AuNP sensors at pH 3.5-9.5. A slight aggregation of sensors in the presence of BPA is observed at $\mathrm{pH}>5.5$ as the concentration of $\mathrm{Ca}^{2+}$ increases to $100 \mu \mathrm{M}$. When the concentration of $\mathrm{Ca}^{2+}$ reaches $50 \mu \mathrm{M}$, aggregation of SRF-AuNP sensors caused by PBP and TBBPA is promoted especially at pH 7.5-9.5 (Fig. 2 and Fig S8, ESI $\dagger$ ). The color of the sensor solutions changes from rose to pink, red or blue (Fig. 2). A heavy aggregation of sensors can be seen in the TEM image after reaction with $\mathrm{PBP}$ in $\mathrm{Ca}^{2+}$ solution at $\mathrm{pH} 7.5$ (Fig. 1B). This result suggests that the influence of $\mathrm{Ca}^{2+}$ on the sorption of BPA and two brominated phenols to NOM can be detected obviously even when the concentration of $\mathrm{Ca}^{2+}$ is as low as $50 \mu \mathrm{M}$ or $100 \mu \mathrm{M}$.

We detect the solution $\mathrm{pH}$ values of the sensor suspension after the sorption of these targets. The fluctuation of $\mathrm{pH}$ is negligible in all cases, indicating that the aggregation of sensors triggered by BPA, PBP and TBBPA is not related to the change of solution $\mathrm{pH}$. Previous studies suggest that the affinity of BPA and halogenated phenols to NOM proceed via van der Waals interactions, $\pi-\pi$ stacking interactions or hydrophobic interactions. ${ }^{2,3}$ For the AuNP based colorimetric assay, the sensors can be linked together by these nonspecific interactions among the sorbed targets or between sorbed targets and ligands on AuNP surface. ${ }^{7}$ In the current study, the surface of SRF-AuNP sensors are highly negative ( $\zeta$-potentials of sensor suspensions range from -17.5 to $-62.2 \mathrm{mV}$ at $\mathrm{pH} 1.6-11$, Fig. S9, ESI $\dagger$ ). The repulsive interactions between the negatively

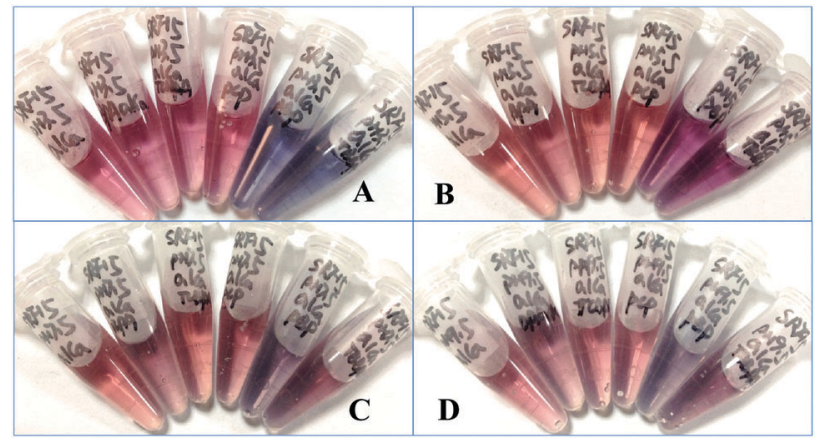

Fig. 2 Photograph of SRF-AuNP suspension in the presence of targets with $0.1 \mathrm{mM} \mathrm{Ca}^{2+}$ at $\mathrm{pH} 3.5$ (A), pH 5.5 (B), pH 7.5 (C), pH 9.5 (D), from left to right: control, BPA, TCBPA, PCP, PBP, and TBBPA, concentration of targets: $10 \mu \mathrm{M}$.

charged oxygen-containing groups of NOM will prevent the aggregation of sensors in water if the affinity of targets can not decrease the density of the negative charges of SRF-AuNPs. On the other hand, the behavior of SRF-AuNP sensors in acid and $\mathrm{Ca}^{2+}$ solution indicates that the interactions with functional groups of NOM can trigger the aggregation of sensors. Therefore, we deduce that sensor aggregation is caused by specific interactions between the target and the oxygen-containing groups of NOM.

The molecular structures of SRF and targets are listed in Fig. 3. The lack of response of sensors to PCP and TCBPA suggests the absence of specific interactions with NOM. To verify the interaction mechanisms between the selected targets with NOM, SRF-AuNPs before and after interaction with these targets are analyzed using XPS. In the O 1s XPS peaks of sensors with the sorbed phenols, the intensities of $\mathrm{C}-\mathrm{OH}$ species increase and the sorbed $\mathrm{H}_{2} \mathrm{O}$ decrease obviously, suggesting the strong sorption of these hydrophobic targets to SRF-AuNP sensors (Fig. 4A, O 1s peaks of BPA, PCP and PBP sorbed sensors are shown in Fig. S10, ESI $\dagger$ ). Compared with the O 1s

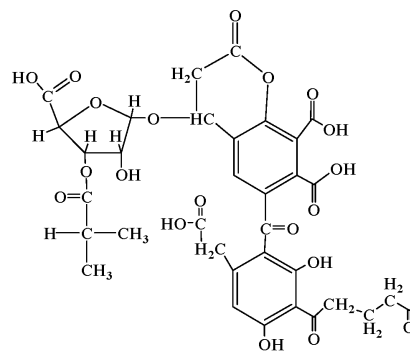

Phthalic acid model of SRF, from ref. 8

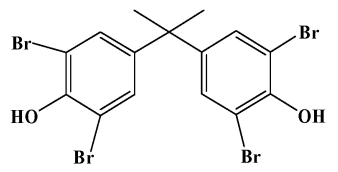

TBBPA, $\mathrm{pKal}=7.5$, $\mathrm{pKa} 2=8.5$, from ref. $2 \mathrm{e}$

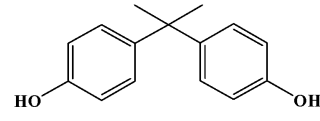

BPA, $\mathrm{pKa}=9.6-10.3$, from ref. 3<smiles>CC(C)(c1cc(Cl)c(O)c(Cl)c1)c1cc(Cl)c(O)c(Cl)c1</smiles>

TCBPA, $\mathrm{pKa}=6.42$, from ref. 9<smiles>Oc1c(Cl)c(Cl)c(Cl)c(Cl)c1Cl</smiles>

PCP, $\mathrm{pKa}=4.35$, from ref. 10<smiles>Oc1c(Br)c(Br)c(Br)c(Br)c1Br</smiles>

PBP, $\mathrm{pKa}=4.4$, from ref. 11
Fig. 3 Molecular structure of Suwannee River fulvic acid and selected targets. 


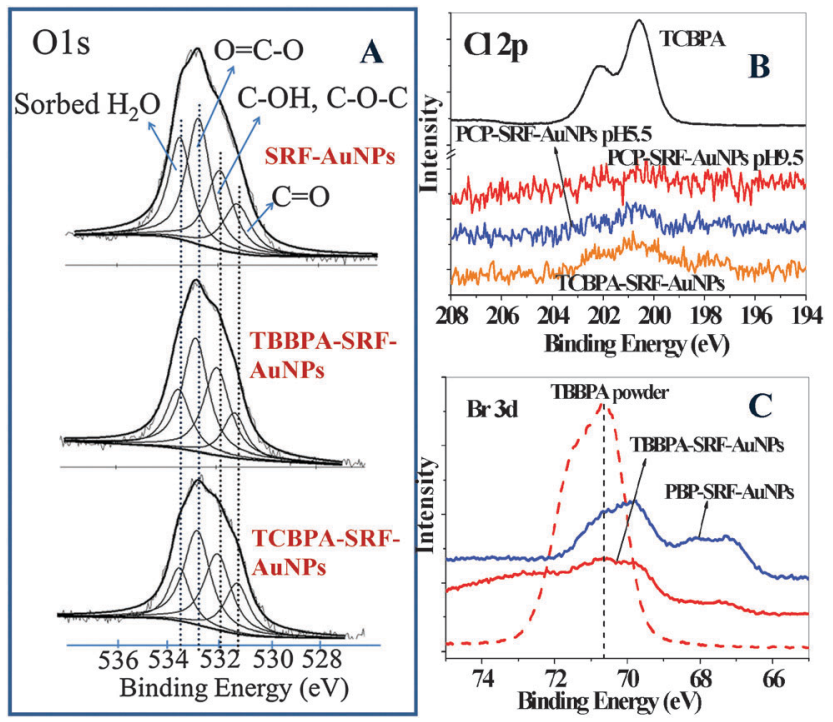

Fig. 4 Fitted XPS O 1s spectra of the as-prepared SRF-AuNP sensors and sensors after reaction with TBBPA and TCBPA at $\mathrm{pH} 5.5$ (A), $\mathrm{Cl} 2 \mathrm{p}$ spectra of TCBPA powder and TCBPA and PCP sorbed SRF-AuNP sensors (B), $\mathrm{Br} 3 \mathrm{~d}$ core-level lines of TBBPA and TBBPA and PBP sorbed SRF-AuNP sensors at $\mathrm{pH} 5.5(\mathrm{C})$, concentration of targets and $\mathrm{Ca}^{2+}: 10 \mu \mathrm{M}$ and $0.1 \mathrm{mM}$, respectively.

lines of the as-prepared AuNP sensors and $\mathrm{Cl} 2 \mathrm{p}$ lines of TCBPA powder, the binding energies of $\mathrm{O}=\mathrm{C}-\mathrm{O}$ and $\mathrm{C}=\mathrm{O}$ species and $\mathrm{C}-\mathrm{Cl}$ bonds do not shift (Fig. 4A and $\mathrm{B}$ ), indicating that the oxidation state of these species is unchanged during sorption, that is, neither the phenolic groups nor the $\mathrm{Cl}$ atoms in their molecules bind with the polar moieties of NOM.

The aggregation of SRF-AuNP sensors in acid solution caused by PBP and TBBPA implies the possible specific interactions between the brominated phenols and NOM. With the increase of solution $\mathrm{pH}$, more O-containing groups of NOM deprotonate, increasing the negative charges of NOM. The phenolic groups of PBP $\left(\mathrm{p} K_{\mathrm{a}}=4.4\right)$ and TBBPA $\left(\mathrm{p} K_{\mathrm{a} 1}=7.5\right.$, $\left.\mathrm{p} K_{\mathrm{a} 2}=8.5\right)$ begin to deprotonate at $\mathrm{pH}>4.4$ and 7.5, respectively. Thereby, the sensors show no response to the two targets at $\mathrm{pH} \geq 7.5$ due to the strengthened electrostatic repulsion between anionic brominated phenols and NOM. $\mathrm{Ca}^{2+}$ cations can chelate negatively charged carboxylic and phenolic groups of NOM, and the value of $\zeta$-potentials of sensors at pH 6.5-11 increases clearly in $100 \mu \mathrm{M}$ of $\mathrm{Ca}^{2+}$ (Fig. S9, ESI $\dagger$ ). The reduced repulsive interactions between the negatively charged targets and sensors facilitate the sorption of targets on the surface of sensors in neutral and alkaline solutions. Therefore, the response of the sensors to PBP and TBBPA is regained at pH 7.5-9.5.

The formation of hydrogen bonds (H-bonds) between these brominated phenols and NOM can be ruled out, because the H-bonds are absent between the corresponding chlorinated phenols and SRF. In the O 1s core-level lines of sensors sorbed with $\mathrm{PBP}$ and TBBPA, the binding energies (BEs) of the $\mathrm{C}=\mathrm{O}$ and $\mathrm{O}=\mathrm{C}-\mathrm{O}$ species show a clear upshift, while the $\mathrm{Br}-\mathrm{C}$ bonds in the $\mathrm{Br} 3 \mathrm{~d}$ signal shift to lower BEs (Fig. $4 \mathrm{~A}$ and $\mathrm{C}$ ). These results imply that the $\mathrm{Br}$ atoms in brominated phenols might bind the polar moieties of NOM, and the former and latter serve as Lewis acid and Lewis base, respectively. We deduce that halogen bonding (XB), a non-covalent interaction, occurs between the $\mathrm{Br}$ atoms and carbonyl and carboxylic groups of NOM by forming $\mathrm{C}-\mathrm{Br} \cdots \mathrm{O}=\mathrm{C}$ bonds, the existence of which has been reported by Auffinger and De Moliner in protein and nucleic acid structures. ${ }^{12}$ Since most XB is unstable in water, this reaction may only occur between sorbed NOM on solids (such as soil and minerals) and brominated phenols. The halogen bonds of charged systems are stronger than those of neutral ones; ${ }^{13}$ therefore, aggregation of SRF-AuNP sensors caused by the two targets is still significant at $\mathrm{pH}$ 7.5-9.5.

To further confirm the specific interactions between brominated phenols and NOM, we detect the response of SRF-AuNP sensors to monobromophenol (4-BP), dibromophenol (DBP), and tribromophenol (TBP). In $0.1 \mathrm{mM}$ of $\mathrm{Ca}^{2+}$ solution, the three phenols cannot trigger the aggregation of sensors at $\mathrm{pH} 3.5$ (Fig. 5A). The aggregation of sensors caused by TBP, DBP and 4-BP begins at $\mathrm{pH} 5.5$, 7.5 and 9.5, respectively (Fig. 5B-D). This result shows that the response of SRF-AuNP sensors to brominated phenols is related to the number of substituted $\mathrm{Br}$ atoms in molecules. Fig. 5 also proves that the formation of $\mathrm{XB}$ between brominated phenols and NOM is more favorable at higher solution $\mathrm{pH}$.

In an acid solution, the absence of response of sensors to BPA implies that BPA affinity towards NOM takes place via nonspecific interactions. In neutral and weakly alkaline solutions, the slight aggregation of SRF-AuNP sensors caused by BPA ( $K_{\mathrm{a}}=$ 9.6-10.3) in the presence of $\mathrm{Ca}^{2+}$ suggests the existence of specific interactions (H-bonds) between BPA and polar groups of NOM, which can be confirmed by the BEs shift of the $\mathrm{O}=\mathrm{C}-\mathrm{O}$ species in the $\mathrm{O}$ 1s spectra of BPA sorbed sensors (Fig. S10, ESI $\dagger$ ). This result is consistent with the assumption of Zhu et al. that the interactions between BPA and dissolved organics occurs primarily due to $\mathrm{H}$-bonds and are not aromatic interactions in neutral solution. ${ }^{3}$
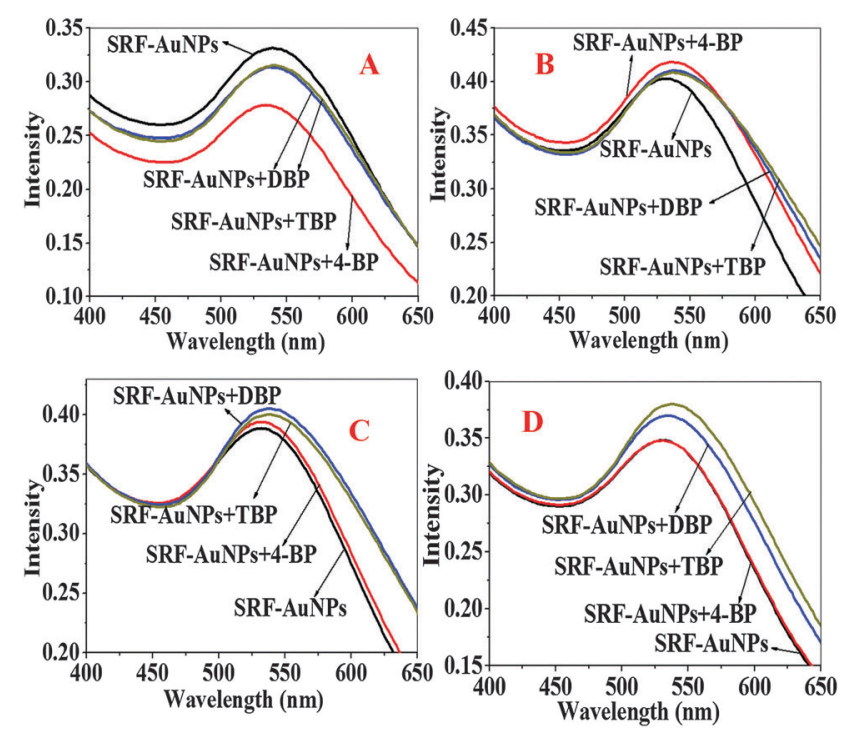

Fig. 5 UV-Vis spectra of the SRF-AuNP suspension in response to 4-BP, DBP and TBP with $0.1 \mathrm{mM} \mathrm{Ca}^{2+}$ at $\mathrm{pH} 3.5$ (A), pH 5.5 (B), pH 7.5 (C), and pH 9.5 (D), concentration of target $10 \mu \mathrm{M}$. 
Suwannee River humic acid (SRH), Pahokee peat humic acid (PPH), Pahokee peat fulvic acid (PPF), and Leonardite humic acid (LH) are also used to modify AuNPs to study the interactions between different kinds of NOM and targets. PPF-AuNP sensors colloids exhibit a red-shift in the presence of BPA, PBP, and TBBPA and no change in PCP and TCBPA. The SRH-AuNP, PPH-AuNP and LH-AuNP sensors respond only to PBP and TBBPA, but the degree of shift of SPR peaks of the three kinds of sensors is less than those of fulvic acid coated sensors (Fig. S11 and S12, ESI $\dagger$ ). The lower sensitivity of the humic acid modified AuNP sensors to their interactions with targets is possibly due to the high aromaticity which provides more nonspecific interactions than specific interactions to targets.

To investigate the sensitivity of the colorimetric assay to the interactions of these targets with NOM, the concentration of targets change in 0.1-100 $\mu \mathrm{M}$. The results show that BPA, TBBPA and PBP respond sensitively to SRF-AuNP sensors, and color changes of the sensor suspension can be observed at BPA, PBP, and TBBPA concentrations as low as 1.0, 1.0 and 0.5 $\mu \mathrm{M}$, respectively (Fig. S13 and S14, ESI $\dagger$ ). The sensors show no response to PCP and TCBPA as their concentrations range in 0.1-100 $\mu \mathrm{M}$.

In summary, we have established a new method to study the possible interaction modes between organic pollutants and NOM using colorimetric assays based on NOM modified AuNPs. The interactions between NOM and targets can be "observed" sensitively according to the color change of AuNP colloids. Through the colorimetric assays, the effect of solution $\mathrm{pH}$, coexisting cations, molecular structure of targets, and NOM aromaticity to organic pollutant affinity to NOM is detected sensitively. For the first time, we find the possibility of the existence of halogen bonds between NOM and brominated phenols. Overall, the colorimetric assays based on AuNP sensors may be a potential alternative to monitor interactions, especially the specific interactions (including $\mathrm{H}$-bonds, halogen-bonds, cation binding) between targets and ligand on the AuNP surface.
We thank the National Basic Research Program of China (2014CB441102), the National High Technology Research and Development Program of China (2013AA065201), the National Natural Science Foundation of China (21537004, 21477140, 21321004), and the Strategic Priority Research Program of the Chinese Academy of Sciences (XDB01020300) for financial support.

\section{Notes and references}

1 W. Chen, L. Duan, L. L. Wang and D. Q. Zhu, Environ. Sci. Technol., 2008, 42, 6862.

2 (a) Y. H. Fei, X. D. Li and X. Y. Li, Mar. Pollut. Bull., 2011, 63, 578; (b) C. Tian, J. T. Wang and X. L. Song, Water, Air, Soil Pollut., 2009, 199, 301; (c) Z. H. Sun, Y. J. Yu, L. Mao, Z. Feng and H. X. Yu, J. Hazard. Mater., 2008, 160, 456; (d) F. D. Paolis and J. Kukkonen, Chemosphere, 1997, 34(8), 1693; (e) W. Han, L. Luo and S. Z. Zhang, Colloids Surf., A, 2013, 428, 60.

3 F.-D. Zhu, K.-H. Choo, H.-S. Chang and B. Lee, Chemosphere, 2012, 87, 857.

4 (a) J. J. Deng, Q. Jiang, Y. X. Wang, L. F. Yang, P. Yu and L. Q. Mao, Anal. Chem., 2013, 85, 9409; (b) Z. H. Zeng, S. Mizukami and K. Kikuchi, Anal. Chem., 2012, 84, 9089; (c) J. J. Deng, P. Yu, Y. X. Wang, L. F. Yang and L. Q. Mao, Adv. Mater., 2014, 26, 6933; (d) M. Zhang, Y. Q. Liu and B. C. Ye, Chem. Commun., 2011, 47, 11849; (e) C.-J. Kim, D.-I. Lee, C. Kim, K. Lee, C.-H. Lee and I.-S. Ahn, Anal. Chem., 2014, 86, 3825.

5 X. Liu, M. Atwater, J. H. Wang and Q. Hou, Colloids Surf., B, 2007, 58, 3.

6 O. Furman, S. Usenko and B. L. T. Lau, Environ. Sci. Technol., 2013, 47, 1349.

7 (a) H. Y. Niu, S. H. Wang, Z. Zhou, Y. R. Ma, X. F. Ma and Y. Q. Cai, Anal. Chem., 2014, 86, 4170; (b) T. Shirman, T. Arad and M. E. van der Boom, Angew. Chem., Int. Ed., 2009, 48, 1.

8 E. A. Nantsis and W. R. Carper, THEOCHEM, 1998, 468, 51.

9 P. M. Bastos, Comparison of experimentally and theoretically determined oxidation and photochemical transformation rates of some organohalogens to promote prediction of persistence, $\mathrm{PhD}$ dissertation, Stockholm University, Stockholm, Sweden, 2009.

10 L. P. Luo, F. X. Liu, Q. K. Yue, F. Chen, Q. Yang, B. L. Hu and Y. X. Chen, Appl. Geochem., 2013, 33, 76.

11 H. Kuramochi, K. Maeda and K. Kawamoto, Environ. Toxicol. Chem., 2004, 23(6), 1386.

12 (a) P. Auffinger, F. A. Hays, E. Westhof and P. Shing Ho, Proc. Natl. Acad. Sci. U. S. A., 2004, 101(48), 16789; (b) E. De Moliner, N. R. Brown and L. N. Johnson, Eur. J. Biochem., 2003, 270, 3174.

13 A. C. C. Carlsson, A. X. Veiga and M. Erdélyi, Top. Curr. Chem., 2015, $359,49$. 